\title{
Transcriptomic analysis of a gut epithelial cell line after knockdown of the selenoprotein glutathione peroxidase 4
}

\author{
P. Cole-Ezea ${ }^{1}$, G. Gong ${ }^{1}$, D. Swan ${ }^{1}$, D. Shanley ${ }^{2}$ and J. Hesketh ${ }^{1}$ \\ ${ }^{1}$ Institute for Cell and Molecular Biosciences and Human Nutrition Research Centre and ${ }^{2}$ Institute for Ageing and Health, \\ Faculty of Medical Sciences, Newcastle University, Framlington Place, Newcastle-upon-Tyne NE2 4HH, UK
}

Selenium, a micronutrient essential for human health, is present in selenoproteins including glutathione peroxidases (GPx) 1-4. Selenium supplementation has been reported to lower mortality from colorectal cancer (CRC) ${ }^{(1)}$ and a common SNP in the GPx4 gene has been reported to influence the risk of $\mathrm{CRC}^{(2)}$. GPx4 exists as cytosolic and mitochondrial isoforms which protect cells from damaging free radicals, particularly lipid hydroperoxides, and may also influence apoptosis ${ }^{(3)}$. The aim of the current study was to study the role of GPx4 in gut epithelial cells using small interference RNA (siRNA) to knockdown GPx4 expression in Caco-2 cells and then carry out a transcriptomic analysis. After 3 days of incubation with a specific siRNA designed to lower expression of both cytosolic and mitochondrial isoforms, GPx4 expression was knocked down by approximately $80 \%$ compared to cells treated with a scrambled control, as assessed by Western blotting. Total RNA was extracted from cells treated for $3 \mathrm{~d}$ with either the GPx4-specific siRNA or the scrambled control and global gene expression analysed by hybridisation to whole genome Illumina HumanHT-12v3 microarrays. Data analysis was performed using the Rank Product package (BioConductor) and Ingenuity Pathway Analysis (IPA). A total of 796 genes showed a 1.2-fold or greater change in gene expression with $P<0.05$. Pathway analysis highlighted changes in pathways associated with mitochondrial dysfunction, oxidative phosphorylation, ubiquinine biosynthesis and oxidative stress. More detailed examination of the transcriptomic data indicated alterations in the expression of genes encoding components of oxidative phosphorylation complexes I, V and IV as well as downregulation of the mitochondrial apoptosis inducing factor (AIF). DNA fragmentation was observed in cells treated with the GPx4-specific siRNA, indicating apoptosis. These data suggest that a knockdown of GPx4 in Caco-2 cells causes alterations in mitochondrial and apoptotic function and these targets are being confirmed by real-time PCR, Western blotting to assess levels of protein components of complex 1 and functional assays such as respirometry.

PC-E was supported by an EPSRC studentship.

1. Whanger PD (2004) Selenium and its relationship to cancer: an update. Br J Nutr 91, 11-28.

2. Bermano G, Pagmantidis V, Holloway N et al. (2007) Evidence that a polymorphism within the $3^{\prime}$-UTR of glutathione peroxidase 4 is functional and is associated with susceptibility to colorectal cancer. Gene Nutr 10, 225-232.

3. Ran Q, Liang H, Gu M et al. (2004) Transgenic mice overexpressing glutathione peroxidase 4 are protected against oxidative stress-induced apoptosis. $J$ Biol Chem 279, 55137-55146. 